\title{
A Flexible Extension to an Extreme Distribution
}

\author{
Mohamed S. Eliwa ${ }^{1}$ (D), Fahad Sameer Alshammari ${ }^{2}$, Khadijah M. Abualnaja ${ }^{3}$ and Mahmoud El-Morshedy ${ }^{2,4, *}$ \\ 1 Department of Mathematics, Faculty of Science, Mansoura University, Mansoura 35516, Egypt; \\ mseliwa@mans.edu.eg \\ 2 Department of Mathematics, College of Science and Humanities in Al-Kharj, \\ Prince Sattam bin Abdulaziz University, Al-Kharj 11942, Saudi Arabia; f.alshammari@psau.edu.sa \\ 3 Department of Mathematics and Statistics, College of Science, Taif University, P.O. Box 11099, \\ Taif 21944, Saudi Arabia; Kh.abualnaja@tu.edu.sa \\ 4 Department of Statistics, Faculty of Science, Mansoura University, Mansoura 35516, Egypt \\ * Correspondence: m.elmorshedy@psau.edu.sa
}

check for

updates

Citation: Eliwa, M.S.; Alshammari, F.S.; Abualnaja, K.M.; El-Morshedy, M. A Flexible Extension to an Extreme Distribution. Symmetry 2021, 13, 745. https://doi.org/10.3390/sym13050745

Academic Editor: Zhivorad Tomovski

Received: 16 March 2021

Accepted: 19 April 2021

Published: 23 April 2021

Publisher's Note: MDPI stays neutral with regard to jurisdictional claims in published maps and institutional affiliations.

Copyright: (C) 2021 by the authors Licensee MDPI, Basel, Switzerland. This article is an open access article distributed under the terms and conditions of the Creative Commons Attribution (CC BY) license (https:/ / creativecommons.org/licenses/by/ $4.0 /)$.

\begin{abstract}
The aim of this paper is not only to propose a new extreme distribution, but also to show that the new extreme model can be used as an alternative to well-known distributions in the literature to model various kinds of datasets in different fields. Several of its statistical properties are explored. It is found that the new extreme model can be utilized for modeling both asymmetric and symmetric datasets, which suffer from over- and under-dispersed phenomena. Moreover, the hazard rate function can be constant, increasing, increasing-constant, or unimodal shaped. The maximum likelihood method is used to estimate the model parameters based on complete and censored samples. Finally, a significant amount of simulations was conducted along with real data applications to illustrate the use of the new extreme distribution.
\end{abstract}

Keywords: probability distributions; skewed and symmetric data; maximum likelihood estimation; hazard rate function; censored samples

\section{Introduction}

Data analysis has become of great interest in many fields of science such as health sciences, reliability analysis, industry, environmental studies, and others. The requirement of obtaining suitable models and statistical distributions has become essential, since defining new distributions will enable us to better describe and predict phenomenal and experimental data. See for example [1-9], among others.

Recently, several methods of obtaining new distributions from old ones have been developed. Many generalized classes of life time distributions have been discussed in the literature. It has been proven in many papers that the new generalizations are more flexible in modelling and better fit real-life data. These new distributions also have several desirable properties such as the asymptotic behavior of their probability density function and the hazard rate function's monotonicity, which has made them superior to the original distribution. All of this has encouraged authors to work more on developing new lifetime distributions using different generalization methods. Here, we refer to the papers of [10] for the Marshall-Olkin class, [11] for the Beta and Gamma classes, [12] for the odd exponentiated half-logistic-G (OEHL-G) family, [13] for the flexible Weibull class, [14] for the odd log-logistic Lindley class, [15] for the odd Chen class, [16] for the exponentiated odd Chen class, [17] for a new Kumaraswamy generalized class, [18,19] for the extended Gamma and log-Bilal models, respectively, [20] for type I half logistic odd Weibull-G and [21] for the Poisson transmuted-G family, among others. 
Here, we use the OEHL-G family of distributions to build a new flexible model with three parameters. The cumulative distribution function (CDF) of the OEHL-G family with two positive shape parameters $\alpha$ and $\lambda$ can be reported as

$$
\Pi(x ; \lambda, \boldsymbol{\Theta}, \alpha)=\left\{\frac{1-e^{\frac{-\lambda G(x ; \boldsymbol{\Theta})}{1-G(x ; \boldsymbol{\Theta})}}}{1+e^{\frac{-\lambda G(x ; \boldsymbol{\Theta})}{1-G(x ; \boldsymbol{\Theta})}}}\right\}^{\alpha} ; x \in \aleph \subset \mathbb{R}
$$

where $G(x ; \boldsymbol{\Theta})$ is the CDF of the baseline distribution under consideration (for more details, see Afify et al., 2017). In our study, the baseline CDF is the inverse exponential (IEx) distribution. The IEx model can be utilized to model datasets which have inverted bathtub failure rates (see, [22]), but it lacks model datasets that are highly skewed "asymmetric" (see, [23]). Therefore, it is essential to have a skewness property in the IEx distribution so that it would be able to fit asymmetry in the datasets that are heavily skewed. Hence, our goal is to obtain a generalized distribution of the IEx model such that it will extend the IEx distribution and also add more flexible features to this life-time model. Many authors have proposed some inverted models due to their flexibility in modeling various types of datasets in different fields (for instance, $[24,25]$ ). The basic motivations for using the odd exponentiated half-logistic inverse exponential (OEHLIEx) distribution in practice are the following:

1. The PDF, CDF, and HRF can be derived in closed-forms.

2. The HRF can be constant, increasing, increasing-constant or unimodal shaped, which makes the proposed model able to be used to analyze different types of datasets.

3. It can be used to model both symmetry and asymmetric datasets.

4. It has more flexibility as compared to well-known models, especially the IEx model.

5. It can be used to model platykurtic-shaped data.

6. It can be used to model over- and under-dispersed data (See Section 6).

\section{The OEHLIEx Distribution}

A random variable $X$ is said to have the IEx distribution with parameter $\beta$ if its CDF $G(x ; \beta)=e^{\frac{-\beta}{x}}$. Using the CDF of the IEx model in Equation (1), we obtain the CDF of the OEHLIEx distribution, which can be expressed as

$$
F(x ; \lambda, \beta, \alpha)=\left\{1-e^{-\lambda\left(e^{\frac{\beta}{x}}-1\right)^{-1}}\right\}^{\alpha}\left\{1+e^{-\lambda\left(e^{\frac{\beta}{x}}-1\right)^{-1}}\right\}^{-\alpha} ; x>0,
$$

where $\alpha$ and $\lambda$ are the positive shape parameters, while $\beta$ is the positive scale parameter. The corresponding probability density function (PDF) to Equation (2) is

$$
f(x ; \lambda, \beta, \alpha)=2 \alpha \lambda \beta x^{-2} e^{-\frac{\beta}{x}} e^{-\lambda\left(e^{\frac{\beta}{x}}-1\right)^{-1}}\left\{1-e^{-\frac{\beta}{x}}\right\}^{-2}\left\{1-e^{-\lambda\left(e^{\frac{\beta}{x}}-1\right)^{-1}}\right\}^{\alpha-1}\left\{1+e^{-\lambda\left(e^{\frac{\beta}{x}}-1\right)^{-1}}\right\}^{-\alpha-1} .
$$

The PDF of the OEHLIEx model can be represented as an infinite mixture of an exponentiated IEx (Exp-IEx) distribution:

$$
f(x ; \lambda, \beta, \alpha)=\sum_{k, l=0}^{\infty} \varphi_{k, l} \tau_{k+l+1}(x),
$$

where

$$
\varphi_{k, l}=2 \alpha \lambda \sum_{j, i=0}^{\infty} \frac{(-1)^{j+k+l}(\lambda(i+j+1))^{k}}{k !(k+l+1)}\left(\begin{array}{c}
-\alpha-1 \\
i
\end{array}\right)\left(\begin{array}{c}
\alpha-1 \\
j
\end{array}\right)\left(\begin{array}{c}
-k-2 \\
l
\end{array}\right)
$$

and

$$
\tau_{k+l+1}(x)=\frac{(k+l+1) \beta}{x^{2}} e^{-\frac{(k+l+1) \beta}{x}},
$$


represents the Exp-IEx density with the power parameter $(k+l+1)$. The corresponding reliability function to Equation (2) can be obtained as

$$
R(x ; \lambda, \beta, \alpha)=1-\left\{1-e^{-\lambda\left(e^{\frac{\beta}{x}}-1\right)^{-1}}\right\}^{\alpha}\left\{1+e^{-\lambda\left(e^{\frac{\beta}{x}}-1\right)^{-1}}\right\}^{-\alpha} ; x>0 .
$$

For the proposed model, the hazard rate function (HRF) and its cumulative can be reported, respectively, as

$$
h(x ; \lambda, \beta, \alpha)=\frac{\left.2 \alpha \lambda \beta x^{-2} e^{-\frac{\beta}{x}}\left\{1-e^{-\frac{\beta}{x}}\right\}^{-2}\left\{1-e^{-\lambda\left(e^{\frac{\beta}{x}}-1\right)^{-1}}\right\}^{\alpha-1}\left\{1+e^{-\lambda\left(e^{\frac{\beta}{x}}-1\right)}\right\}^{-1}\right\}^{-1}}{e^{\lambda\left(e^{\frac{\beta}{x}}-1\right)^{-1}}\left(\left\{1+e^{-\lambda\left(e^{\frac{\beta}{x}}-1\right)^{-1}}\right\}^{\alpha}-\left\{1-e^{-\lambda\left(e^{\frac{\beta}{x}}-1\right)}{ }^{-1}\right\}\right)}
$$

and

$$
H(x ; \lambda, \beta, \alpha)=-\log \left(1-\left\{1-e^{-\lambda\left(e^{\frac{\beta}{x}}-1\right)}\right\}^{-1}\left\{1+e^{-\lambda\left(e^{\frac{\beta}{x}}-1\right)^{-1}}\right\}^{-\alpha}\right),
$$

where the cumulative hazard function is the integral of the hazard function. Figure 1 shows the PDF plots and its HRF for different parameter values. It is immediate that the PDF is unimodal shaped and can be used to discuss right- and left-skewed datasets, whereas the HRF can be increasing, constant, increasing-constant, or unimodal shaped. Therefore, the OEHLIEx distribution can be utilized to analyze various types of data in several practical fields.
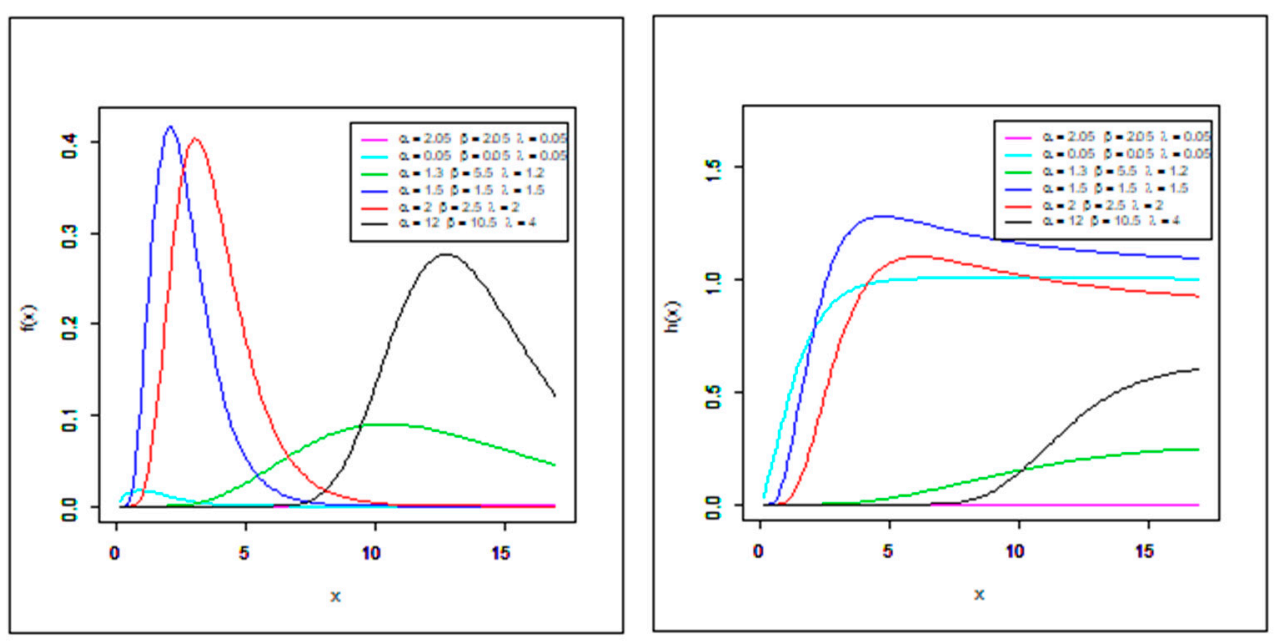

Figure 1. The PDF "left panel" and HRF "right panel" plots.

\section{Statistical and Reliability Properties}

\subsection{Quantile Function (QF), Skewness, and Kurtosis}

The QF of the OEHLIEx distribution is given as follows: if $U$ has a uniform random variable on $U(0,1)$, then

$$
X_{U}=-\beta\left\{\ln \left(\frac{-\ln \left(1-u^{\frac{1}{\alpha}}\right)+\ln \left(1+u^{\frac{1}{\alpha}}\right)}{\lambda-\ln \left(1-u^{\frac{1}{\alpha}}\right)+\ln \left(1+u^{\frac{1}{\alpha}}\right)}\right)\right\}^{-1} .
$$

Equation (8) can be used to generate a random sample, and the median can be derived at $u=0.5$. Moreover, it can be used to obtain the skewness and kurtosis, where skewness $=\frac{X_{3 / 4}+X_{1 / 4}-2 X_{2 / 4}}{X_{3 / 4}-X_{1 / 4}}$ and Kurtosis $=\frac{X_{3 / 8}-X_{1 / 8}+X_{7 / 8}-X_{5 / 8}}{X_{6 / 8}-X_{2 / 8}}$. Tables 1-3 report 
some numerical values of the quantiles, skewness, and kurtosis of the OEHLIEx model using Maple software.

Table 1. The quantiles, skewness, and kurtosis for $\lambda \rightarrow \infty$.

\begin{tabular}{|c|c|c|c|c|c|c|c|c|}
\hline \multicolumn{3}{|c|}{ Parameter $\downarrow$ Measure $\rightarrow$} & \multirow{2}{*}{$X_{1 / 4}$} & \multirow{2}{*}{$X_{2 / 4}$} & \multirow{2}{*}{$X_{3 / 8}$} & \multirow{2}{*}{$X_{5 / 8}$} & \multirow{2}{*}{ Skewness } & \multirow{2}{*}{ Kurtosis } \\
\hline$\alpha$ & $\beta$ & $\lambda$ & & & & & & \\
\hline \multirow{6}{*}{0.5} & \multirow{6}{*}{0.5} & 0.5 & 0.311 & 0.733 & 0.491 & 1.055 & 0.296 & 1.268 \\
\hline & & 0.9 & 0.238 & 0.492 & 0.349 & 0.678 & 0.270 & 1.263 \\
\hline & & 2.0 & 0.177 & 0.314 & 0.239 & 0.406 & 0.221 & 1.246 \\
\hline & & 3.5 & 0.149 & 0.243 & 0.193 & 0.302 & 0.181 & 1.229 \\
\hline & & 100 & 0.075 & 0.095 & 0.085 & 0.104 & -0.007 & 1.183 \\
\hline & & 150 & 0.071 & 0.088 & 0.079 & 0.596 & -0.021 & 1.181 \\
\hline
\end{tabular}

Table 2. The quantiles, skewness, and kurtosis for $\beta \rightarrow \infty$.

\begin{tabular}{|c|c|c|c|c|c|c|c|c|}
\hline \multicolumn{3}{|c|}{ Parameter $\downarrow$ Measure $\rightarrow$} & \multirow{2}{*}{$X_{1 / 4}$} & \multirow{2}{*}{$X_{2 / 4}$} & \multirow{2}{*}{$X_{3 / 8}$} & \multirow{2}{*}{$X_{5 / 8}$} & \multirow{2}{*}{ Skewness } & \multirow{2}{*}{ Kurtosis } \\
\hline$\alpha$ & $\lambda$ & $\beta$ & & & & & & \\
\hline \multirow{6}{*}{0.3} & \multirow{6}{*}{1.5} & 0.2 & 0.046 & 0.093 & 0.066 & 0.132 & 0.345 & 1.348 \\
\hline & & 0.8 & 0.184 & 0.373 & 0.264 & 0.529 & 0.345 & 1.348 \\
\hline & & 1.5 & 0.345 & 0.699 & 0.495 & 0.991 & 0.345 & 1.348 \\
\hline & & 5.0 & 1.150 & 2.332 & 1.649 & 3.305 & 0.345 & 1.348 \\
\hline & & 10 & 2.301 & 4.664 & 3.299 & 6.609 & 0.345 & 1.348 \\
\hline & & 100 & 23.008 & 46.639 & 32.995 & 66.095 & 0.345 & 1.348 \\
\hline
\end{tabular}

Table 3. The quantiles, skewness, and kurtosis for $\alpha \rightarrow \infty$.

\begin{tabular}{|c|c|c|c|c|c|c|c|c|}
\hline \multicolumn{3}{|c|}{ Parameter $\downarrow$ Measure $\rightarrow$} & \multirow{2}{*}{$X_{1 / 4}$} & \multirow{2}{*}{$X_{2 / 4}$} & \multirow{2}{*}{$X_{3 / 8}$} & \multirow{2}{*}{$X_{5 / 8}$} & \multirow{2}{*}{ Skewness } & \multirow{2}{*}{ Kurtosis } \\
\hline$\beta$ & $\lambda$ & $\alpha$ & & & & & & \\
\hline \multirow{6}{*}{1.5} & \multirow{6}{*}{2.0} & 0.02 & 0.00 & 0.00 & 0.00 & 0.063 & 1.000 & 1.548 \\
\hline & & 0.05 & 0.00 & 0.108 & 0.076 & 0.159 & 0.169 & 1.784 \\
\hline & & 0.1 & 0.108 & 0.216 & 0.153 & 0.318 & 0.464 & 1.808 \\
\hline & & 0.2 & 0.216 & 0.429 & 0.305 & 0.615 & 0.393 & 1.457 \\
\hline & & 0.8 & 0.795 & 1.279 & 1.027 & 1.572 & 0.162 & 1.242 \\
\hline & & 1.5 & 1.231 & 1.756 & 1.491 & 2.056 & 0.127 & 1.259 \\
\hline
\end{tabular}

Regarding Tables $1-3$, the proposed model is suitable for modelling asymmetric as well as symmetric datasets that are platykurtic shaped.

\subsection{Incomplete Moments}

The sth incomplete moments can be listed as

$$
\omega_{\mathcal{S}}(t)=\sum_{k, l=0}^{\infty} \varphi_{k, l} \omega_{S}^{*}(t)
$$


where $\omega_{s}^{*}(t)=\int_{0}^{t} x^{s} \tau_{k+l+1}(x) d x$. Thus, the sth incomplete moments of the OEHLIEx model can be proposed as

$$
\omega_{s}(t)=\sum_{k, l, n=0}^{\infty} \varphi_{k, l} \frac{\{-\beta(k+l+1)\}^{n}}{n !(s-n-1)} t^{s-n-1}
$$

3.3. Reliability Function of Linear Consecutive, Parallel, Series, and Bridge Type Network Systems

If the random variable $X$ has the OEHLIEx distribution, then the reliability function of the linear consecutive $k$ - out - of $n: F$ system can be expressed as

$$
\begin{aligned}
R_{L}(t ; k, n) & =\sum_{j=0}^{m} N(j ; k, n)\left\{R^{n-j}(t) F(t)\right\}^{j} \\
& =\sum_{j, i=0}^{m}(-1)^{j}\left(\begin{array}{l}
j \\
i
\end{array}\right) N(j ; k, n) R^{n-j+i}(t)
\end{aligned}
$$

$$
=\sum_{j, i=0}^{m}(-1)^{j}\left(\begin{array}{l}
j \\
i
\end{array}\right) N(j ; k, n)\left\{1-\left\{1-e^{-\lambda\left(e^{\frac{\beta}{x}}-1\right)^{-1}}\right\}^{\alpha}\left\{1+e^{-\lambda\left(e^{\frac{\beta}{x}}-1\right)^{-1}}\right\}^{-\alpha}\right\}^{n-j+i}
$$

for more details concerning the values of $m, N(j ; k, n)$, and $j$, see [26]. In the special case of the system $k-o u t-o f-n: F$, the parallel and series system when $k=n$ and $k=1$, respectively.

Consider two systems: one of them is parallel, whereas the other is a series with independent $n$ components. Each component has the OEHLIEx model; thus, the reliability function in the case of the parallel system can be reported as

$$
R_{P-S}(x)=1-\left[\left\{1-e^{-\lambda\left(e^{\frac{\beta}{x}}-1\right)^{-1}}\right\}^{\alpha}\left\{1+e^{-\lambda\left(e^{\frac{\beta}{x}}-1\right)^{-1}}\right\}^{-\alpha}\right]^{n},
$$

where as the reliability function in the case of the series system can be expressed as

$$
R_{S-S}(x)=\left[1-\left\{1-e^{-\lambda\left(e^{\frac{\beta}{x}}-1\right)^{-1}}\right\}^{\alpha}\left\{1+e^{-\lambda\left(e^{\frac{\beta}{x}}-1\right)^{-1}}\right\}^{-\alpha}\right]^{n} .
$$

In reliability theory, there exists another type of engineering system in the so-called bridge-type network or a complex system, which has many applications in this field. Such systems as these can be evaluated by using many approaches such as conditional probability, a connection matrix, and tree diagrams, as well as cut and tie sets. Assume a bridge-type network consists of five components (A, B, C, D, and E) where each component has the OEHLIEx model, these components can be connected as follows:

Consider the previous network in which success requires that at least one of the paths AC, BD, AED, or BEC is good. To evaluate the reliability function of this network, the conditional probability approach has been utilized. The previous network in Figure 2 can be subdivided into two systems, one with E considered bad, i.e., it always failed, and one with $\mathrm{E}$ considered good, i.e., it cannot fail. Thus,

$$
\begin{gathered}
R_{N W}=R_{N W}(\text { if E is good }) R_{E}(x ; \lambda, \beta, \alpha)+R_{N W}(\text { if E is bad }) F_{E}(x ; \lambda, \beta, \alpha) \\
=\left\{\left(1-F_{A}(x ; \lambda, \beta, \alpha) F_{B}(x ; \lambda, \beta, \alpha)\right)\left(1-F_{C}(x ; \lambda, \beta, \alpha) F_{D}(x ; \lambda, \beta, \alpha)\right)\right\} R_{E}(x ; \lambda, \beta, \alpha) \\
+\left\{1-\left(1-R_{A}(x ; \lambda, \beta, \alpha) R_{C}(x ; \lambda, \beta, \alpha)\right)\left(1-R_{A}(x ; \lambda, \beta, \alpha) R_{D}(x ; \lambda, \beta, \alpha)\right)\right\} F_{E}(x ; \lambda, \beta, \alpha),
\end{gathered}
$$

where $F_{*}=1-R_{*}$ represents the unreliability function of a component $\left(^{*}\right)$. Since $R_{A}=$ $R_{B}=R_{C}=R_{D}=R_{E}$, then the reliability $R_{N W}$ can be expressed as

$$
R_{N W}=2\left\{R(x ; \lambda, \beta, \alpha)^{2}+R(x ; \lambda, \beta, \alpha)^{3}+R(x ; \lambda, \beta, \alpha)^{5}\right\}-5 R(x ; \lambda, \beta, \alpha)^{4} .
$$




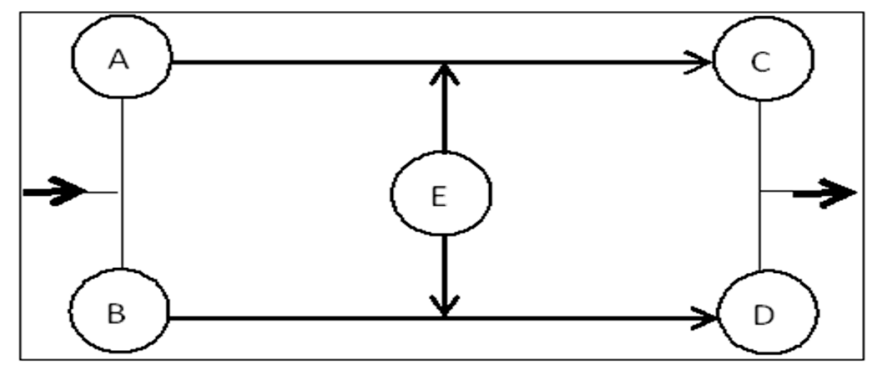

Figure 2. Bridge-type network.

Assume four different systems, namely, parallel, serious, linear consecutive, and bridge network; each system consists of 20 components, except the bridge network which consists of five components. Tables 4-6 list some numerical values of the reliability function for these systems using Maple software.

Table 4. Some numerical values of the reliability for different systems for various values of $\alpha$.

\begin{tabular}{|c|c|c|c|c|c|c|}
\hline \multirow{2}{*}{$\begin{array}{c}\text { System } \downarrow \\
\text { Parameter } \rightarrow\end{array}$} & \multicolumn{2}{|c|}{$\alpha=1.5$} & \multicolumn{2}{|c|}{$\alpha=5$} & \multicolumn{2}{|c|}{$\alpha=10$} \\
\hline & $\beta=0.5$ & $\lambda=0.5$ & $\beta=0.5$ & $\lambda=0.5$ & $\beta=0.5$ & $\lambda=0.5$ \\
\hline Parallel & \multicolumn{2}{|c|}{0.4036} & \multicolumn{2}{|c|}{0.8215} & \multicolumn{2}{|c|}{0.9681} \\
\hline Series & \multicolumn{2}{|c|}{$1.4 \times 10^{-32}$} & \multicolumn{2}{|c|}{$2.2 \times 10^{-22}$} & \multicolumn{2}{|c|}{$9.7 \times 10^{-17}$} \\
\hline Linear Consecutive & \multicolumn{2}{|c|}{0.0157} & \multicolumn{2}{|c|}{0.0179} & \multicolumn{2}{|c|}{0.0967} \\
\hline Bridge Network & \multicolumn{2}{|c|}{0.00133} & \multicolumn{2}{|c|}{0.01453} & \multicolumn{2}{|c|}{0.0551} \\
\hline
\end{tabular}

Table 5. Some numerical values of the reliability for different systems for various values of $\beta$.

\begin{tabular}{|c|c|c|c|}
\hline System $\downarrow$ & $\beta=0.5$ & $\beta=0.8$ & $\beta=1.5$ \\
\hline Parameter $\rightarrow$ & $\lambda=0.1$ & $\lambda=0.1$ & $\alpha=0.1$ \\
\hline Parallel & 0.8041 & 0.9213 & 0.9798 \\
\hline Series & $7.5 \times 10^{-23}$ & $3.5 \times 10^{-19}$ & $9.5 \times 10^{-16}$ \\
\hline Linear Consecutive & 0.0597 & 0.0689 & 0.0794 \\
\hline Bridge Network & 0.0130 & 0.0309 & 0.0695 \\
\hline
\end{tabular}

Table 6. Some numerical values of the reliability for different systems for various values of $\lambda$.

\begin{tabular}{|c|c|c|c|c|c|c|}
\hline \multirow{2}{*}{$\begin{array}{c}\text { System } \downarrow \\
\text { Parameter } \rightarrow\end{array}$} & \multicolumn{2}{|c|}{$\lambda=0.3$} & \multicolumn{2}{|c|}{$\lambda=0.6$} & \multicolumn{2}{|c|}{$\lambda=0.9$} \\
\hline & $\alpha=0.8$ & $\beta=0.7$ & $\alpha=0.8$ & $\beta=0.7$ & $\alpha=0.8$ & $\beta=0.7$ \\
\hline Parallel & \multicolumn{2}{|c|}{0.9873} & \multicolumn{2}{|c|}{0.4459} & \multicolumn{2}{|c|}{0.0771} \\
\hline Series & \multicolumn{2}{|c|}{$7.3 \times 10^{-15}$} & \multicolumn{2}{|c|}{$1.8 \times 10^{-31}$} & \multicolumn{2}{|c|}{$1.1 \times 10^{-48}$} \\
\hline Linear Consecutive & \multicolumn{2}{|c|}{0.0567} & \multicolumn{2}{|c|}{0.0426} & \multicolumn{2}{|c|}{0.0411} \\
\hline Bridge Network & \multicolumn{2}{|c|}{0.0855} & \multicolumn{2}{|c|}{0.0017} & \multicolumn{2}{|c|}{$3.2 \times 10^{-6}$} \\
\hline
\end{tabular}

Regarding Tables 4-6, it is clear that the reliability of parallel, series, linear consecutive, and bridge network systems increases in two cases, one of them for fixed values of $\beta$ and $\lambda$ with $\alpha \rightarrow \infty$, and the other for fixed values of $\alpha$ and $\lambda$ with $\beta \rightarrow \infty$. Whereas, the reliability of these systems decreases for fixed values of $\alpha$ and $\beta$ with $\lambda \rightarrow \infty$. 


\subsection{Entropy}

The Rényi entropy of a positive random variable $X$ representing a measure of variation of the uncertainty is defined by

$$
\begin{aligned}
I_{\rho}(X) & =\frac{1}{1-\rho} \log \left(\int_{0}^{\infty} f^{\rho}(x ; \lambda, \beta, \alpha) d x\right) ; \rho>0 \text { and } \rho \neq 1 . \\
& =\frac{1}{1-\rho} \log \left(\sum_{k, l=0}^{\infty} \Omega_{k, l} \int_{0}^{\infty} g^{\rho}(x) G^{k+l}(x) d x\right) \\
& =\frac{1}{1-\rho} \log \left(\sum_{k, l=0}^{\infty} \Omega_{k, l} \frac{\beta^{1-\rho} \Gamma(2 \rho-1)}{(\rho+k+l)^{2 \rho-1}}\right),
\end{aligned}
$$

where $(2 \rho-1) \neq-1,-2,-3, \ldots$ and

$$
\Omega_{k, l}=(2 \alpha \lambda)^{\rho} \sum_{j, i=0}^{\infty} \frac{(-1)^{j+k+l}(\lambda(i+j+1))^{k}}{k !}\left(\begin{array}{c}
-\alpha \rho-\rho \\
i
\end{array}\right)\left(\begin{array}{c}
\rho \alpha-\rho \\
j
\end{array}\right)\left(\begin{array}{c}
-k-2 \rho \\
l
\end{array}\right) .
$$

The binomial coefficients will be computed with the negative values using built-in

\begin{tabular}{|c|c|c|c|c|c|c|}
\hline \multirow{2}{*}{ Entropy $\downarrow$ Parameter $\rightarrow$} & \multicolumn{2}{|c|}{$\beta=0.5$} & \multicolumn{2}{|c|}{$\beta=0.8$} & \multicolumn{2}{|c|}{$\beta=1.5$} \\
\hline & $\alpha=0.1$ & $\lambda=0.1$ & $\alpha=0.1$ & $\lambda=0.1$ & $\alpha=0.1$ & $\lambda=0.1$ \\
\hline Renyi & \multicolumn{2}{|c|}{0.2569} & \multicolumn{2}{|c|}{0.3697} & \multicolumn{2}{|c|}{0.4302} \\
\hline Shannon & \multicolumn{2}{|c|}{0.1987} & \multicolumn{2}{|c|}{0.1997} & \multicolumn{2}{|c|}{0.2159} \\
\hline
\end{tabular}
functions in Maple software. The Shannon entropy is a special case of the Rényi entropy when $\rho \rightarrow 1$. Tables 7-9 list some numerical values of entropies using Maple software.

\begin{tabular}{|c|c|c|c|c|c|c|}
\hline \multirow{2}{*}{ Entropy $\downarrow$ Parameter $\rightarrow$} & \multicolumn{2}{|c|}{$\alpha=1.5$} & \multicolumn{2}{|c|}{$\alpha=5$} & \multicolumn{2}{|c|}{$\alpha=10$} \\
\hline & $\beta=0.5$ & $\lambda=0.5$ & $\beta=0.5$ & $\lambda=0.5$ & $\beta=0.5$ & $\lambda=0.5$ \\
\hline Renyi & \multicolumn{2}{|c|}{2.3694} & \multicolumn{2}{|c|}{2.5976} & \multicolumn{2}{|c|}{3.6871} \\
\hline Shannon & \multicolumn{2}{|c|}{2.5364} & \multicolumn{2}{|c|}{2.6974} & \multicolumn{2}{|c|}{2.7639} \\
\hline
\end{tabular}

Table 7. Entropies for different values of $\beta$.

\begin{tabular}{|c|c|c|c|c|c|c|}
\hline \multirow{2}{*}{ Entropy $\downarrow$ Parameter $\rightarrow$} & \multicolumn{2}{|c|}{$\lambda=0.3$} & \multicolumn{2}{|c|}{$\lambda=0.6$} & \multicolumn{2}{|c|}{$\lambda=0.9$} \\
\hline & $\alpha=0.8$ & $\beta=0.7$ & $\alpha=0.8$ & $\beta=0.7$ & $\alpha=0.8$ & $\beta=0.7$ \\
\hline Renyi & \multicolumn{2}{|c|}{3.2168} & \multicolumn{2}{|c|}{3.0197} & \multicolumn{2}{|c|}{2.4398} \\
\hline Shannon & \multicolumn{2}{|c|}{3.1020} & \multicolumn{2}{|c|}{3.009} & \multicolumn{2}{|c|}{2.9578} \\
\hline
\end{tabular}

Table 8. Entropies for different values of $\alpha$.

Table 9. Entropies for different values of $\lambda$.

Regarding Tables 7-9, it is clear that the entropy increases in two cases, one of them for fixed values of $\alpha$ and $\lambda$ with $\beta \rightarrow \infty$, and the other for fixed values of $\beta$ and $\lambda$ with $\alpha \rightarrow \infty$. Whereas, the entropy decreases for fixed values of $\alpha$ and $\beta$ with $\lambda \rightarrow \infty$. 


\section{Parameters Estimation}

\subsection{Maximum Likelihood Estimation (MLE) Based on Complete Samples.}

In this section, we derive the MLE of the unknown parameters $\lambda, \beta$, and $\alpha$ of the OEHLIEx model based on complete samples. Consider a random sample $X_{1}, X_{2}, \ldots, X_{n}$ from the OEHLIEx model; then, the log-likelihood $(L L)$ function can be expressed as

$$
L=\sum_{i=1}^{n} \log f\left(x_{i} ; \lambda, \beta, \alpha\right) .
$$

By substituting from Equation (3) into Equation (17), the MLEs of the OEHLIEx parameters can be obtained by maximizing

$$
\begin{aligned}
L L= & n \log 2 \alpha \lambda \beta-2 \sum_{i=1}^{n} \log x_{i}-\beta \sum_{i=1}^{n} \frac{1}{x_{i}}-\lambda \sum_{i=1}^{n}\left(e^{\frac{\beta}{x_{i}}}-1\right)^{-1}-2 \sum_{i=1}^{n} \log \left(1-e^{-\frac{\beta}{x_{i}}}\right)+ \\
& (\alpha-1) \sum_{i=1}^{n} \log \left(1-e^{-\lambda\left(e^{\frac{\beta}{x_{i}}}-1\right)}-(\alpha+1) \sum_{i=1}^{n} \log \left(1+e^{-\lambda\left(e^{\frac{\beta}{x_{i}}}-1\right)}-1\right),\right.
\end{aligned}
$$

with respect to $\lambda, \beta$, and $\alpha$. We used $\mathbf{R}$ software to obtain the parameters' values. The $(1-\delta)$ $100 \%$ confidence intervals (CIs) of the model parameters can be calculated using the following relations:

$$
\hat{\lambda} \pm Z_{\frac{\delta}{2}} \sqrt{\operatorname{var}(\hat{\lambda})}, \hat{\beta} \pm Z_{\frac{\delta}{2}} \sqrt{\operatorname{var}(\hat{\beta})} \text { and } \hat{\alpha} \pm Z_{\frac{\delta}{2}} \sqrt{\operatorname{var}(\hat{\alpha})},
$$

where $Z_{\frac{\delta}{2}}$ is the upper $\left(\frac{\delta}{2}\right)$ th percentile of the standard normal distribution.

\subsection{MLE Based on Type-II Censored Samples}

The likelihood function for a type-II censored sample can be reported as

$$
l=\frac{n !}{(n-k) !}\left(R\left(x_{k}\right)\right)^{n-k} \prod_{i=1}^{k} f\left(x_{i}\right),
$$

where $n$ represents the number of components, and the experiment is stopped when $k$ items failed. If $X_{1}, X_{2}, \ldots, X_{n}$ represent an independent and identically distributed random sample from the OEHLIEx distribution and $X_{1}, X_{2}, \ldots, X_{k}, k \leq n$ represent an ordered sample obtained from a type-II right censoring sample, then the log-likelihood $\left(L L^{*}\right)$ function is

$$
L L^{*}=\ln \frac{n !}{(n-k) !}+(n-k) \ln R\left(x_{k} ; \lambda, \beta, \alpha\right)+\sum_{i=1}^{k} \log f\left(x_{i} ; \lambda, \beta, \alpha\right) .
$$

By substituting from Equations (3) and (5) into Equation (20), the MLEs of the OEHLIEx parameters can be obtained by maximizing results of the equation with respect to $\lambda, \beta$, and $\alpha$.

\section{Simulation Results}

We assessed the performance of the MLE approach with respect to various samples size. The assessment was based on a simulation study:

1. Generate 1000 samples of size $n=10,20,30,50,75,100,150$ from the OEHLIEx model under various values of the parameters.

2. Compute the MLEs for the 1000 samples, say $\hat{\theta}_{\mathrm{j}}$ for $j=1,2, \ldots, 1000$.

3. Compute the biases and mean-squared errors (MSEs), where

$$
\operatorname{Bias}(\theta)=\frac{1}{1000} \sum_{j=1}^{1000}\left(\hat{\theta}_{j}-\theta\right) \text { and } \operatorname{MSE}(\theta)=\frac{1}{1000} \sum_{j=1}^{1000}\left(\hat{\theta}_{j}-\theta\right)^{2} .
$$


The empirical results for complete data are given in Tables 10-14 using OEHLIEx(1.5, $0.5,0.9)$, OEHLIEx $(2.5,2.5,2.5)$, OEHLIEx $(0.5,0.8,0.9)$, and OEHLIEx $(0.5,0.5,0.5)$, respectively. Whereas the empirical results for type-II censored data when $k=20$ are given in Tables 15-17 for OEHLIEx(0.7,0.8,0.9), OEHLIEx $(0.5,1.5,2.5)$, OEHLIEx $(0.01,0.5,0.1 .5)$, and OEHLIEx $(0.1,1.5,0.5)$, respectively.

Table 10. The bias and MSE (in parentheses) for schema I.

\begin{tabular}{cccccccc}
\hline Parameters $\downarrow$ Sample Size $\rightarrow$ & $\mathbf{1 0}$ & $\mathbf{2 0}$ & $\mathbf{3 0}$ & $\mathbf{5 0}$ & $\mathbf{7 5}$ & $\mathbf{1 0 0}$ & $\mathbf{1 5 0}$ \\
\hline$\lambda$ & 0.12578 & 0.11467 & 0.11025 & 0.11012 & 0.09987 & 0.04237 & 0.01983 \\
& $(0.15964)$ & $(0.15083)$ & $(0.14998)$ & $(0.14322)$ & $(0.10021)$ & $(0.06867)$ & $(0.01325)$ \\
\hline \multirow{2}{*}{$\beta$} & 0.10361 & 0.10318 & 0.10251 & 0.10112 & 0.09876 & 0.05217 & 0.02649 \\
& $(0.13555)$ & $(0.13200)$ & $(0.11036)$ & $(0.10998)$ & $(0.08655)$ & $(0.03524)$ & $(0.00145)$ \\
\hline \multirow{2}{*}{$\alpha$} & 0.11666 & 0.10008 & 0.09866 & 0.07624 & 0.03654 & 0.01254 & 0.01001 \\
& $(0.12372)$ & $(0.10187)$ & $(0.08873)$ & $(0.05326)$ & $(0.02361)$ & $(0.01023)$ & $(0.00147)$ \\
\hline
\end{tabular}

Table 11. The bias and MSE (in parentheses) for schema II.

\begin{tabular}{cccccccc}
\hline Parameters $\downarrow$ Sample Size $\rightarrow$ & $\mathbf{1 0}$ & $\mathbf{2 0}$ & $\mathbf{3 0}$ & $\mathbf{5 0}$ & $\mathbf{7 5}$ & $\mathbf{1 0 0}$ & $\mathbf{1 5 0}$ \\
\hline$\lambda$ & 0.11769 & 0.10987 & 0.10644 & 0.08657 & 0.05634 & 0.01237 & 0.00558 \\
& $(0.15793)$ & $(0.14026)$ & $(0.13657)$ & $(0.11087)$ & $(0.08741)$ & $(0.03219)$ & $(0.00543)$ \\
\hline \multirow{2}{*}{$\beta$} & 0.10222 & 0.10104 & 0.10057 & 0.08741 & 0.05234 & 0.03201 & 0.00874 \\
& $(0.11236)$ & $(0.10658)$ & $(0.09874)$ & $(0.05324)$ & $(0.01240)$ & $(0.00874)$ & $(0.00149)$ \\
\hline \multirow{2}{*}{$\alpha$} & 0.10984 & 0.10567 & 0.10111 & 0.07876 & 0.05234 & 0.023264 & 0.00951 \\
& $(0.10037)$ & $(0.09759)$ & $(0.05687)$ & $(0.04217)$ & $(0.03217)$ & $(0.01007)$ & $(0.00188)$ \\
\hline
\end{tabular}

Table 12. The bias and MSE (in parentheses) for schema III.

\begin{tabular}{cccccccc}
\hline Parameters $\downarrow$ Sample Size $\rightarrow$ & $\mathbf{1 0}$ & $\mathbf{2 0}$ & $\mathbf{3 0}$ & $\mathbf{5 0}$ & $\mathbf{7 5}$ & $\mathbf{1 0 0}$ & $\mathbf{1 5 0}$ \\
\hline \multirow{2}{*}{$\lambda$} & 0.10998 & 0.10756 & 0.10564 & 0.102341 & 0.08764 & 0.035418 & 0.00429 \\
& $(0.13067)$ & $(0.12361)$ & $(0.12248)$ & $(0.10252)$ & $(0.03101)$ & $(0.01097)$ & $(0.00574)$ \\
\hline \multirow{2}{*}{$\beta$} & 0.09987 & 0.09153 & 0.08635 & 0.053243 & 0.022324 & 0.020011 & 0.008214 \\
& $(0.09996)$ & $(0.09864)$ & $(0.05314)$ & $(0.03221)$ & $(0.01002)$ & $(0.00517)$ & $(0.00120)$ \\
\hline \multirow{2}{*}{$\alpha$} & 0.076249 & 0.075324 & 0.063397 & 0.048891 & 0.039217 & 0.011871 & 0.003814 \\
& $(0.057681)$ & $(0.056341)$ & $(0.042224)$ & $(0.033364)$ & $(0.02109)$ & $(0.00996)$ & $(0.00225)$ \\
\hline
\end{tabular}

Table 13. The bias and MSE (in parentheses) for schema IV.

\begin{tabular}{cccccccc}
\hline Parameters $\downarrow$ Sample Size $\rightarrow$ & $\mathbf{1 0}$ & $\mathbf{2 0}$ & $\mathbf{3 0}$ & $\mathbf{5 0}$ & $\mathbf{7 5}$ & $\mathbf{1 0 0}$ & $\mathbf{1 5 0}$ \\
\hline \multirow{2}{*}{$\lambda$} & 0.110542 & 0.100214 & 0.095874 & 0.086554 & 0.045638 & 0.023211 & 0.010014 \\
& $(0.102201)$ & $(0.101123)$ & $(0.082210)$ & $(0.053329)$ & $(0.033415)$ & $(0.00524)$ & $(0.00221)$ \\
\hline \multirow{2}{*}{$\beta$} & 0.085761 & 0.056639 & 0.0355621 & 0.0322624 & 0.020132 & 0.020100 & 0.005624 \\
& $(0.065354)$ & $(0.032544)$ & $(0.023517)$ & $(0.021030)$ & $(0.007461)$ & $(0.00712)$ & $(0.00230)$ \\
\hline \multirow{2}{*}{$\alpha$} & 0.075641 & 0.0655327 & 0.039640 & 0.028634 & 0.019652 & 0.005536 & 0.004187 \\
& $(0.063099)$ & $(0.052201)$ & $(0.044671)$ & $(0.033249)$ & $(0.007956)$ & $(0.004310)$ & $(0.002140)$ \\
\hline
\end{tabular}


Table 14. The bias and MSE (in parentheses) for schema V.

\begin{tabular}{cccccccc}
\hline Parameters $\downarrow$ Sample Size $\rightarrow$ & $\mathbf{1 0}$ & $\mathbf{2 0}$ & $\mathbf{3 0}$ & $\mathbf{5 0}$ & $\mathbf{7 5}$ & $\mathbf{1 0 0}$ & $\mathbf{1 5 0}$ \\
\hline$\lambda$ & 0.12578 & 0.11467 & 0.11025 & 0.11012 & 0.09987 & 0.04237 & 0.01983 \\
& $(0.15964)$ & $(0.15083)$ & $(0.14998)$ & $(0.14322)$ & $(0.10021)$ & $(0.06867)$ & $(0.01325)$ \\
\hline \multirow{2}{*}{$\beta$} & 0.10361 & 0.10318 & 0.10251 & 0.10112 & 0.09876 & 0.05217 & 0.02649 \\
& $(0.13555)$ & $(0.13200)$ & $(0.11036)$ & $(0.10998)$ & $(0.08655)$ & $(0.03524)$ & $(0.00145)$ \\
\hline \multirow{2}{*}{$\alpha$} & 0.11666 & 0.10008 & 0.09866 & 0.07624 & 0.03654 & 0.01254 & 0.01001 \\
& $(0.12372)$ & $(0.10187)$ & $(0.08873)$ & $(0.05326)$ & $(0.02361)$ & $(0.01023)$ & $(0.00147)$ \\
\hline
\end{tabular}

Table 15. The bias and MSE (in parentheses) for VI.

\begin{tabular}{cccccccc}
\hline Parameters $\downarrow$ Sample Size $\rightarrow$ & $\mathbf{1 0}$ & $\mathbf{2 0}$ & $\mathbf{3 0}$ & $\mathbf{5 0}$ & $\mathbf{7 5}$ & $\mathbf{1 0 0}$ & $\mathbf{1 5 0}$ \\
\hline \multirow{2}{*}{$\lambda$} & 0.11458 & 0.11536 & 0.11256 & 0.10123 & 0.08563 & 0.01326 & 0.002366 \\
& $(0.12694)$ & $(0.10083)$ & $(0.09844)$ & $(0.08436)$ & $(0.06369)$ & $(0.03698)$ & $(0.00743)$ \\
\hline \multirow{2}{*}{$\beta$} & 0.11963 & 0.11326 & 0.11269 & 0.10269 & 0.08963 & 0.02301 & 0.00147 \\
& $(0.11538)$ & $(0.10563)$ & $(0.10269)$ & $(0.10111)$ & $(0.07488)$ & $(0.02398)$ & $(0.00789)$ \\
\hline \multirow{2}{*}{$\alpha$} & 0.17896 & 0.16236 & 0.14265 & 0.12488 & 0.08963 & 0.03521 & 0.00723 \\
& $(0.13698)$ & $(0.12856)$ & $(0.10328)$ & $(0.09994)$ & $(0.09602)$ & $(0.05326)$ & $(0.00117)$ \\
\hline
\end{tabular}

Table 16. The bias and MSE (in parentheses) for VII.

\begin{tabular}{cccccccc}
\hline Parameters $\downarrow$ Sample Size $\rightarrow$ & $\mathbf{1 0}$ & $\mathbf{2 0}$ & $\mathbf{3 0}$ & $\mathbf{5 0}$ & $\mathbf{7 5}$ & $\mathbf{1 0 0}$ & $\mathbf{1 5 0}$ \\
\hline \multirow{2}{*}{$\lambda$} & 0.21653 & 0.18856 & 0.16456 & 0.13698 & 0.09856 & 0.05602 & 0.00147 \\
& $(0.19691)$ & $(0.16136)$ & $(0.15452)$ & $(0.14236)$ & $(0.12013)$ & $(0.09695)$ & $(0.01369)$ \\
\hline \multirow{2}{*}{$\beta$} & 0.22985 & 0.21360 & 0.17203 & 0.13695 & 0.08563 & 0.03214 & 0.00983 \\
& $(0.17803)$ & $(0.16636)$ & $(0.14447)$ & $(0.11029)$ & $(0.09301)$ & $(0.01238)$ & $(0.00650)$ \\
\hline \multirow{2}{*}{$\alpha$} & 0.10256 & 0.09362 & 0.07452 & 0.03698 & 0.00968 & 0.00236 & 0.00063 \\
& $(0.09635)$ & $(0.08503)$ & $(0.06416)$ & $(0.03694)$ & $(0.01236)$ & $(0.00632)$ & $(0.00085)$ \\
\hline
\end{tabular}

Table 17. The bias and MSE (in parentheses) for VIII.

\begin{tabular}{cccccccc}
\hline Parameters $\downarrow$ Sample Size $\rightarrow$ & $\mathbf{1 0}$ & $\mathbf{2 0}$ & $\mathbf{3 0}$ & $\mathbf{5 0}$ & $\mathbf{7 5}$ & $\mathbf{1 0 0}$ & $\mathbf{1 5 0}$ \\
\hline \multirow{2}{*}{$\lambda$} & 0.12690 & 0.12369 & 0.11015 & 0.10850 & 0.10369 & 0.06369 & 0.00856 \\
& $(0.11231)$ & $(0.11197)$ & $(0.11017)$ & $(0.10856)$ & $(0.10285)$ & $(0.07458)$ & $(0.00469)$ \\
\hline \multirow{2}{*}{$\beta$} & 0.043028 & 0.030258 & 0.022136 & 0.014369 & 0.010236 & 0.008896 & 0.001539 \\
& $(0.040695)$ & $(0.03312)$ & $(0.02635)$ & $(0.01802)$ & $(0.01132)$ & $(0.008309)$ & $(0.00239)$ \\
\hline \multirow{2}{*}{$\alpha$} & 0.119980 & 0.11743 & 0.08830 & 0.05237 & 0.02138 & 0.00856 & 0.00038 \\
& $(0.15891)$ & $(0.13264)$ & $(0.10239)$ & $(0.09721)$ & $(0.07128)$ & $(0.02598)$ & $(0.00653)$ \\
\hline
\end{tabular}

From Tables 10-17, we can say that the MLE approach can be used effectively to estimate the model parameters for both a small and large sample size. This due to the consistency properties of the estimators when $n$ grows.

\section{Data Analysis}

\subsection{Data Analysis and Discussion Based on Complete Samples}

In this section, we illustrate the empirical importance of the OEHLIEx distribution using three applications on real data. These data are used to compare the fits of the OEHLIEx distribution with some competitive models such as the inverse exponential (IEx), exponential (Ex), exponentiated half-logistic (EHL), generalized half-logistic (GHL), and normal $(\mathrm{N})$ models. For the comparison of the models, we should use the values of LL, and Kolmogorov-Smirnov (K-S) test with its $p$-value. 
The first data set (I) represents the relief times of twenty patients receiving an analgesic (see, [27]).

The second data set (II) represents the strengths of glass fibers (see, [28]).

The third data set (III) represents the failure times (in minutes) for a sample of 15 electronic components in an accelerated life test (see, [29]).

Tables 18-20 list the MLEs with their corresponding standard errors (in parentheses), and goodness-of-fit $(\mathrm{GoF})$ measures for the datasets.

Table 18. The MLE(s) and GoF statistics for data set I.

\begin{tabular}{|c|c|c|c|c|c|}
\hline \multirow{2}{*}{ Model $\downarrow$ Parameter $\rightarrow$} & \multicolumn{3}{|c|}{ MLE(s) [Std-err] } & \multirow{2}{*}{$-L L$} & \multirow{2}{*}{$\begin{array}{c}\text { K-S } \\
(p-\text { Value })\end{array}$} \\
\hline & $\alpha$ & $\beta$ & $\lambda$ & & \\
\hline OEHLIEx & 18.389 [12.430] & $0.070[0.175]$ & 0.159 [0.399] & 16.327 & $\begin{array}{c}0.134 \\
(0.863)\end{array}$ \\
\hline IEx & $1.724[0.385]$ & - & - & 32.668 & $\begin{array}{c}0.387 \\
(0.005)\end{array}$ \\
\hline Ex & $0.526[0.117]$ & - & - & 32.837 & $\begin{array}{c}0.439 \\
(0.001) \\
\end{array}$ \\
\hline GHL & $0.731[0.163]$ & - & - & 29.464 & $\begin{array}{c}0.398 \\
(0.003)\end{array}$ \\
\hline EHL & $2.780[0.621]$ & - & - & 22.908 & $\begin{array}{c}0.281 \\
(0.085)\end{array}$ \\
\hline $\mathrm{N}$ & $1.90[0.1535]$ & $0.6863[0.1085]$ & - & 20.8498 & $\begin{array}{c}0.2079 \\
(0.3528)\end{array}$ \\
\hline
\end{tabular}

The $(1-\delta) 100 \%$ CIs of the parameters $\alpha, \beta$, and $\lambda$ are, respectively, [0, 42.752], [0, 0.413], and [0, 0.943].

Table 19. The MLE(s) and GoF statistics for data set II.

\begin{tabular}{|c|c|c|c|c|c|}
\hline \multirow{2}{*}{ Model $\downarrow$ Parameter $\rightarrow$} & \multicolumn{3}{|c|}{ MLE(s) [Std-err] } & \multirow{2}{*}{$-L L$} & \multirow{2}{*}{$\begin{array}{c}\text { K-S } \\
(p \text {-Value })\end{array}$} \\
\hline & $\alpha$ & $\beta$ & $\lambda$ & & \\
\hline OEHLIEx & 82.594 [41.246] & $0.013[0.003]$ & $0.047[0.013]$ & 22.602 & $\begin{array}{c}0.071 \\
(0.887)\end{array}$ \\
\hline IEx & $1.526[0.192]$ & - & - & 92.805 & $\begin{array}{c}0.468 \\
(<0.001)\end{array}$ \\
\hline Ex & $0.618[0.078]$ & - & - & 93.222 & $\begin{array}{c}0.472 \\
(<0.001)\end{array}$ \\
\hline GHL & $0.896[0.113]$ & - & - & 82.074 & $\begin{array}{c}0.446 \\
(<0.001)\end{array}$ \\
\hline EHL & $2.278[0.287]$ & - & - & 65.942 & $\begin{array}{c}0.396 \\
(<0.001)\end{array}$ \\
\hline $\mathrm{N}$ & $1.6156[0.0602]$ & $0.4779[0.0426]$ & - & 42.8871 & $\begin{array}{l}0.1774 \\
(0.033)\end{array}$ \\
\hline
\end{tabular}

The $(1-\delta) 100 \%$ CIs of the parameters $\alpha, \beta$, and $\lambda$ are, respectively, [1.754, 163.435], [0.006, 0.020], and [0.021, 0.073]. 
Table 20. The MLE(s) and GoF statistics for data set III.

\begin{tabular}{|c|c|c|c|c|c|}
\hline \multirow{2}{*}{ Model $\downarrow$ Parameter $\rightarrow$} & \multicolumn{3}{|c|}{ MLE(s) [Std-err] } & \multirow{2}{*}{$-L L$} & \multirow{2}{*}{$\begin{array}{c}\text { K-S } \\
(p-\text { Value })\end{array}$} \\
\hline & $\alpha$ & $\beta$ & $\lambda$ & & \\
\hline OEHLIEx & $0.962[0.0435]$ & $2.259[4.711]$ & $0.115[0.231]$ & 63.946 & $\begin{array}{c}0.098 \\
(0.996)\end{array}$ \\
\hline IEx & 9.559 [2.468] & - & - & 69.055 & $\begin{array}{c}0.263 \\
(0.209)\end{array}$ \\
\hline Ex & 0.036 [0.009] & - & - & 64.738 & $\begin{array}{c}0.155 \\
(0.807)\end{array}$ \\
\hline GHL & 0.037 [0.009] & - & - & 64.592 & $\begin{array}{c}0.151 \\
(0.833)\end{array}$ \\
\hline EHL & 28.874 [7.455] & - & - & 367.295 & $\begin{array}{c}0.799 \\
(<0.001)\end{array}$ \\
\hline $\mathrm{N}$ & $27.5467[5.1793]$ & 20.0593 [3.6623] & - & 66.2645 & $\begin{array}{c}0.1897 \\
(0.5885)\end{array}$ \\
\hline
\end{tabular}

The $(1-\delta) 100 \%$ CIs of the parameters $\alpha, \beta$, and $\lambda$ are, respectively, $[0.437,2.410],[0,7.803]$, and $[0,0.694]$.

Regarding Tables 18-20, it is clear that the OEHLIEx model is the best model among all tested models. Regarding data set I, it is noted that the TrIEx and EHL models work quite well besides the OEHLIEx model where $p$-value $>0.05$, but we always search for the most fitting model. Thus, we recommend using the OEHLIEx model to analyze data set I. Similarly, for dataset III, it is found that the TrIEx, IEx, Ex, and GHL models work quite well besides the OEHLIEx model, but we also recommend utilizing the OEHLIEx model to analyze these data.

Figures 3-5 show the empirical estimated CDF "ECDF", probability-probability (PP), and fitted PDF "FPDF" plots for data sets I, II, and III, respectively, which support the results of Tables 18-20. Moreover, it is noted that the datasets plausibly came from the OEHLIEx model.
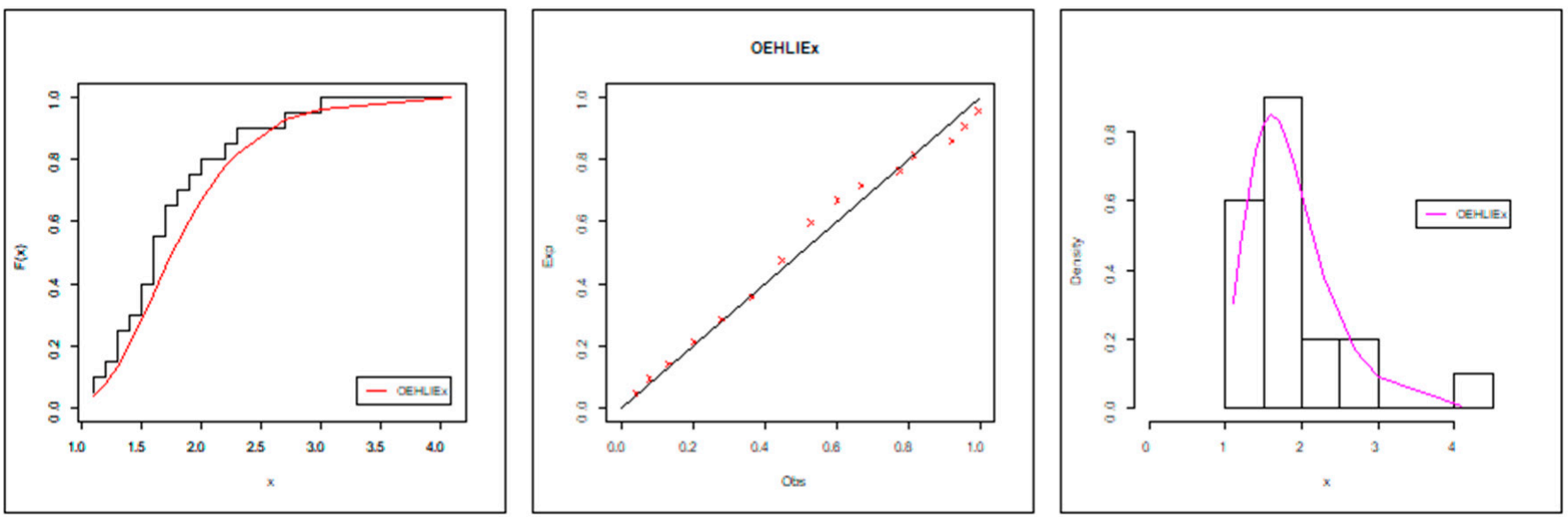

Figure 3. The ECDF "left panel", PP "middle panel", FPDF "right panel" plots for data set I. 

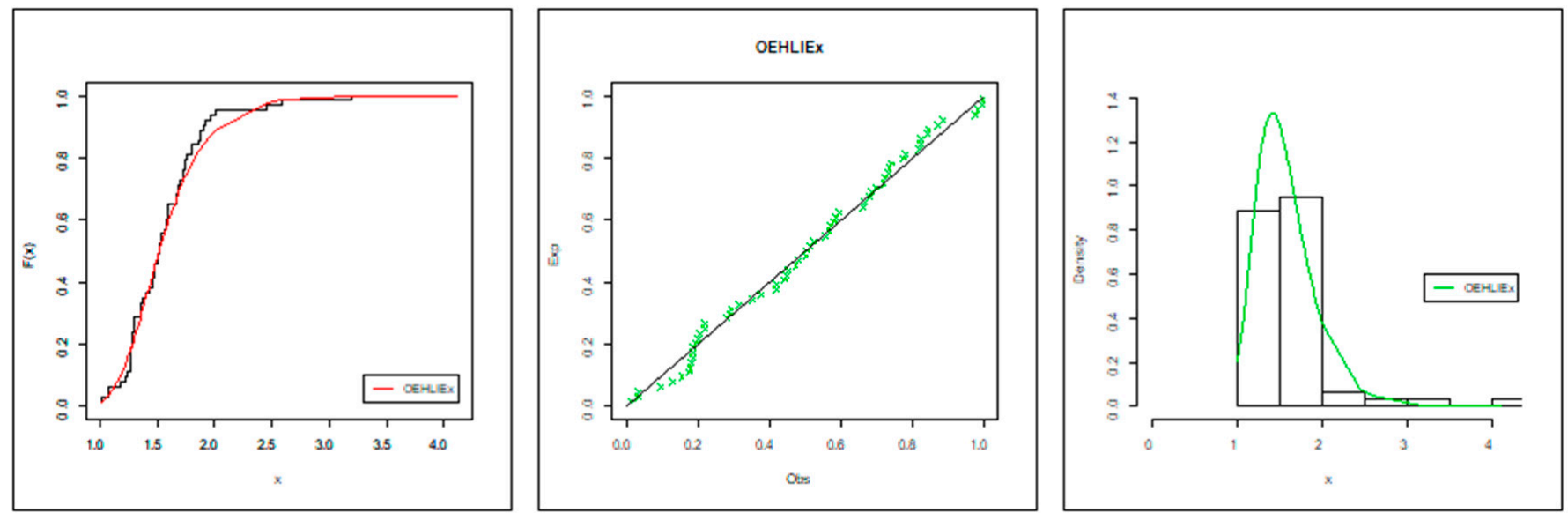

Figure 4. The ECDF "left panel", PP "middle panel", FPDF "right panel" plots for data set II.
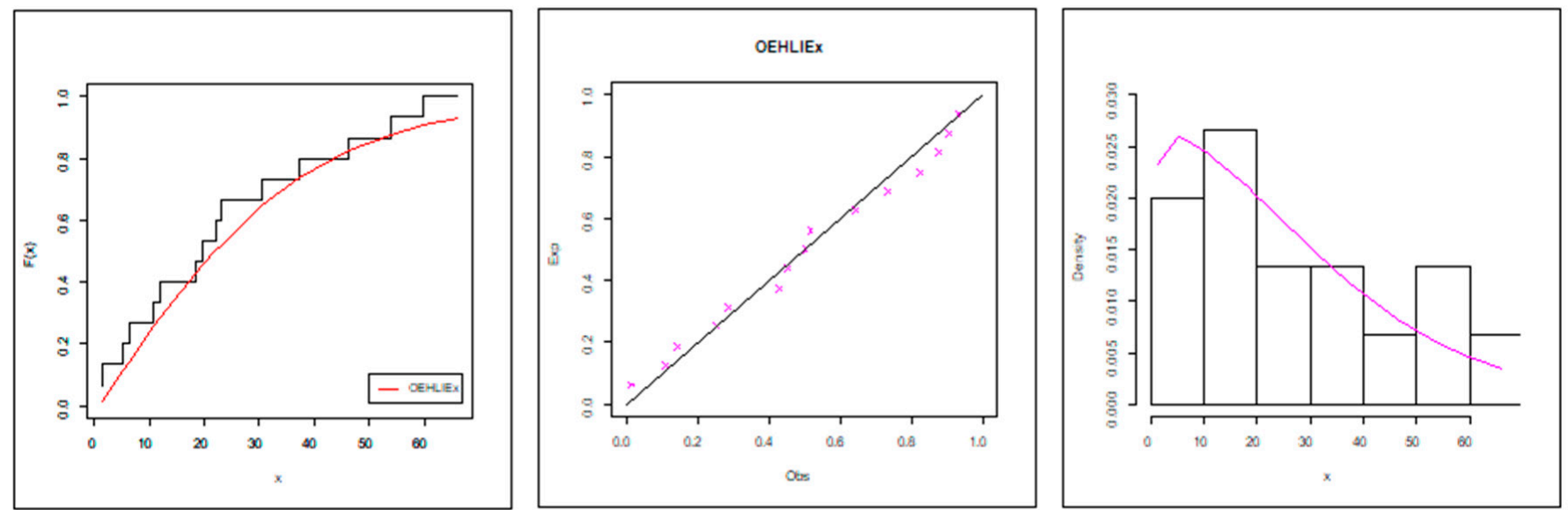

Figure 5. The ECDF "left panel", PP "middle panel", FPDF "right panel" plots for data set III.

Figures 6-8 show the profiles of the LL function "PLLF" based on data sets I, II, and III.
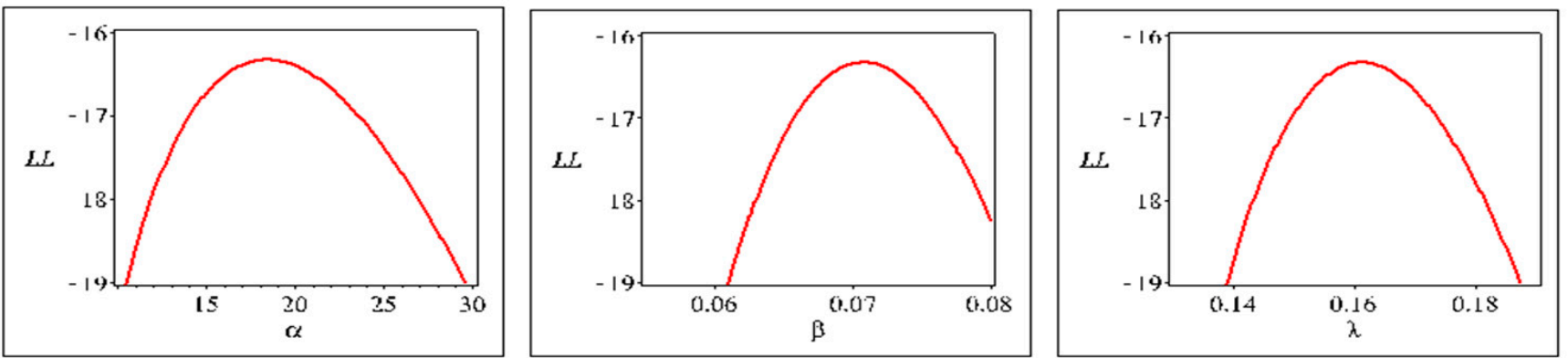

Figure 6. The PLLF for data set I. 

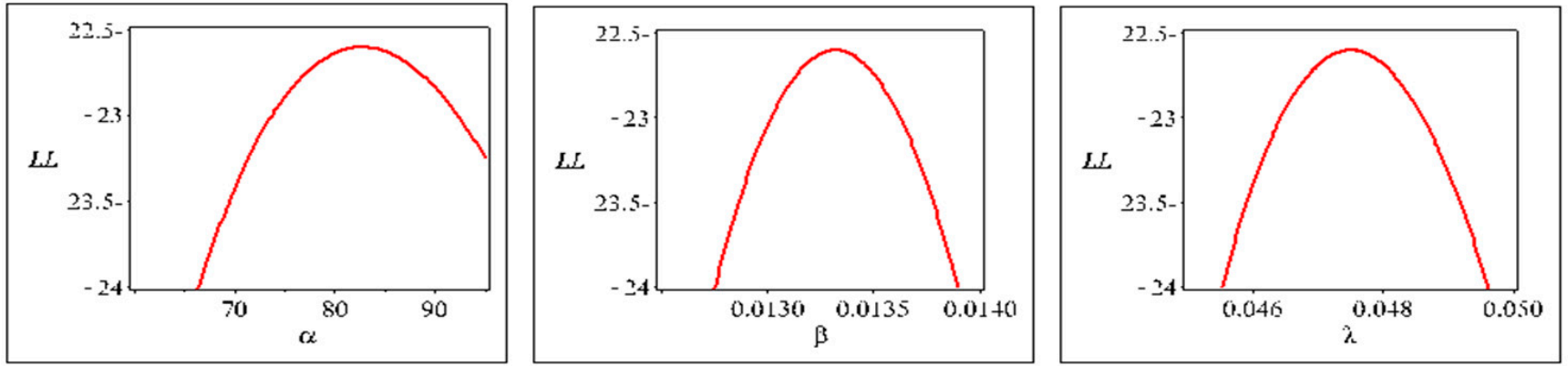

Figure 7. The PLLF for data set II.
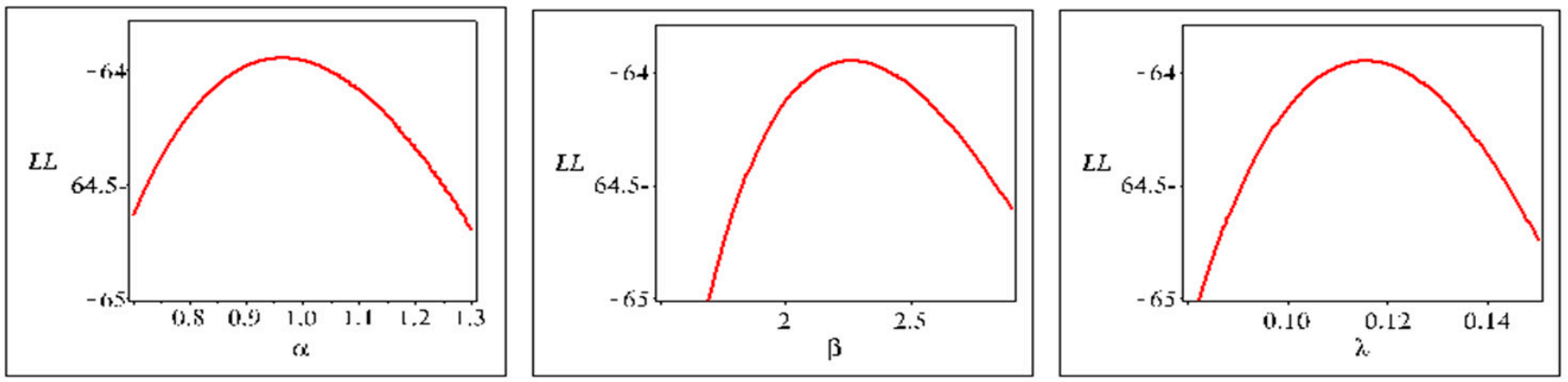

Figure 8. The PLLF for data set III.

Regarding Figures 6-8, it clear that the estimators have a unique solution where the profiles of the LL function are unimodal shaped. Figure 9 shows the total time in test (TTT) plots for data sets I, II, and III. It is clear that the datasets suffer from an increasing hazard rate. Thus, the proposed model can be used to model the HRF for these data sets.
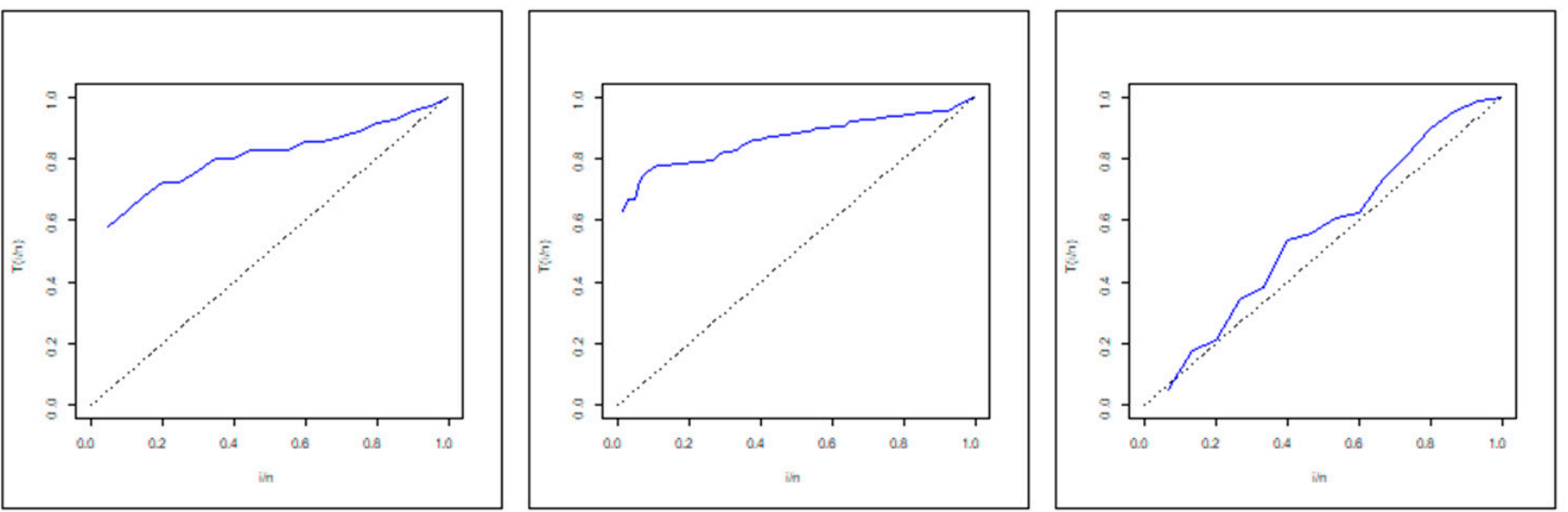

Figure 9. The TTT plots for data I "left panel", data II "middle panel", and data III "right panel".

Table 21 lists some computational statistics for the three datasets by utilizing the OEHLIEx model.

Based on the model parameters, data sets I and II suffer from under-dispersed phenomena (index of dispersion $<1$ ), whereas data set III suffers from over-dispersion (index of dispersion > 1). Moreover, data sets I, II, and III represent positive-skewed data with a platykurtic shape. 
Table 21. Some computational statistics for data sets I, II, and III.

\begin{tabular}{cccccccc}
\hline Data Set & Mean & Variance & Index of Dispersion & Skewness & Kurtosis & Entropy \\
\hline I & 1.900 & 0.496 & 0.261 & 0.119 & 1.278 & 0.756 \\
\hline II & 1.615 & 0.232 & 0.144 & 0.118 & 1.277 & 0.931 \\
\hline III & 27.546 & 431.116 & 15.650 & 0.185 & 1.239 & 1.676 \\
\hline
\end{tabular}

\subsection{Dataset IV: Analysis and Discussion Based on Type-II Right Censored Samples}

The censored data have been obtained from (http:/ /www.biochemia-medica.com, accessed on 6 March 2021). They represent the recovery time of 50 patients suffering from cancer, monthly. The MLEs of the unknown parameters, $-L L^{*}, \mathrm{~K}-\mathrm{S}$, and $p$-value for the proposed model are given in Table 22.

Table 22. The MLE(s) and GoF statistics for data set IV.

\begin{tabular}{|c|c|c|c|c|c|}
\hline \multirow{2}{*}{ Model $\downarrow$ Parameter $\rightarrow$} & \multicolumn{3}{|c|}{ MLE(s) [Std-err] } & \multirow{2}{*}{$-L L^{*}$} & \multirow{2}{*}{$\begin{array}{c}\text { K-S } \\
(p \text {-Value })\end{array}$} \\
\hline & $\alpha$ & $\beta$ & $\beta$ & & \\
\hline OEHLIEx & 131.228 & 0.008 & 0.031 & 17.691 & $\begin{array}{c}0.104 \\
(0.611)\end{array}$ \\
\hline IEx & 1.575 & - & - & 75.162 & $\begin{array}{c}0.463 \\
(<0.001)\end{array}$ \\
\hline Ex & 0.599 & - & - & 75.561 & $\begin{array}{c}0.526 \\
(<0.001) \\
\end{array}$ \\
\hline EHL & 2.388 & - & - & 52.789 & $\begin{array}{c}0.394 \\
(<0.001)\end{array}$ \\
\hline GHL & 0.862 & - & - & 66.682 & $\begin{array}{c}0.501 \\
(<0.001)\end{array}$ \\
\hline
\end{tabular}

Depending on $-L L^{*}, \mathrm{~K}-\mathrm{S}$, and $p$-values, it is noted that the OEHLIEx model is appropriate to analyze data set IV. The $(1-\delta) 100 \%$ CIs of the parameters $\alpha, \beta$, and $\lambda$ are, respectively, [126.999, 137.201], [0, 0.019], and [0, 0.107]. Figure 10 shows the TTT and PP plots based on the type-II right censored sample.
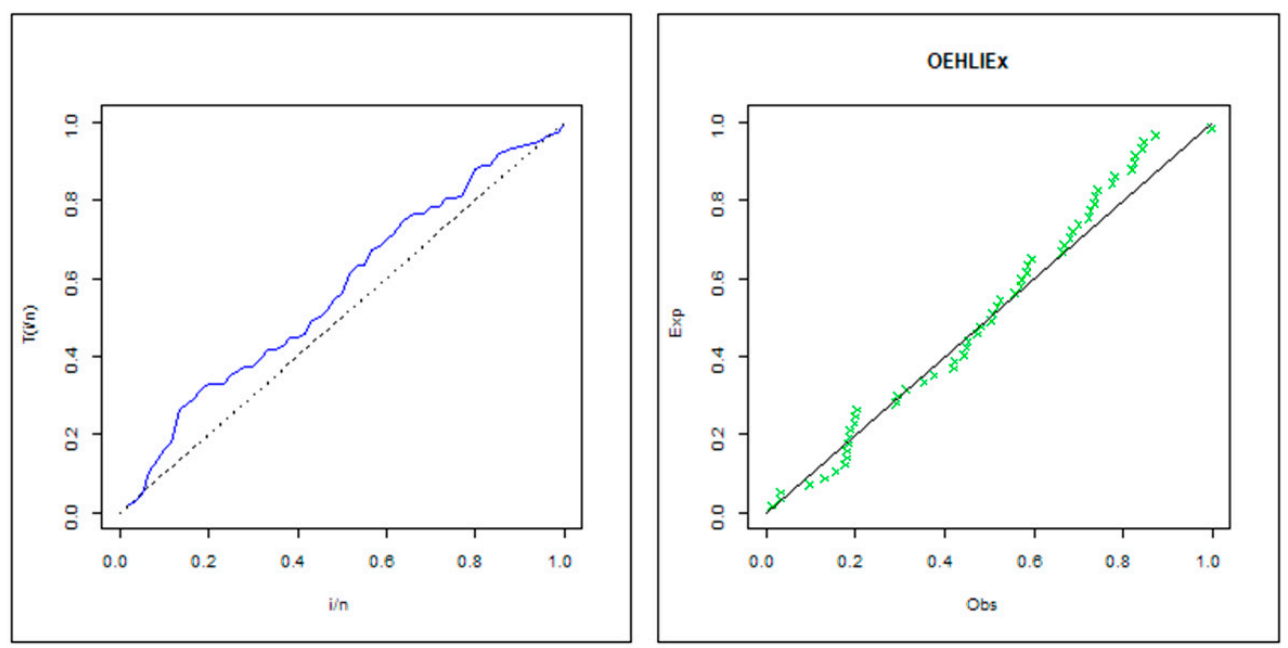

Figure 10. The TTT "left panel" and PP "right panel" plots for data set IV.

According to Figure 10, it is observed that the proposed model fits the data well. Moreover, the shape of the HRF increases. Table 23 shows some computational statistics for data IV utilizing the OEHLIEx model. 
Table 23. Some computational statistics for data set IV.

\begin{tabular}{ccccccc}
\hline Data Set & Mean & Variance & Index of Dispersion & Skewness & Kurtosis & Entropy \\
\hline IV & 1.667 & 0.260 & 0.156 & 0.324 & 1.876 & 1.998 \\
\hline
\end{tabular}

Regarding Table 23, it is noted that the dataset IV suffers from under-dispersed phenomena which are positive skewed and platykurtic shaped.

\section{Conclusions}

In this article, a new flexible extension of an extreme distribution with three-parameter has been proposed, which generalized the inverse exponential distribution. The HRF of the new extension can be constant, increasing, increasing-constant, or unimodal shaped. Furthermore, it can be utilized for modelling asymmetric "positive and negative" as well as symmetric datasets and can be used to model over- and under-dispersed data. Thus, the new extension can be used effectively to model different kinds of data in several fields. The model parameters have been estimated utilizing the MLE approach. A simulation has been performed for different samples sizes, and it was found that the MLE technique works quite well for estimating the parameters for datasets considered herein. Finally, four data applications which illustrate the flexibility of the new extension and its excellence over other models have been also analyzed.

Author Contributions: Conceptualization, M.S.E. and M.E.-M.; methodology, M.S.E. and M.E.-M.; software, M.S.E. and M.E.-M.; validation, F.S.A. and K.M.A.; formal analysis, M.S.E., M.E.-M. and F.S.A.; resources, F.S.A. and K.M.A.; data curation, M.S.E. and M.E.-M.; writing-original draft preparation, M.S.E. and M.E.-M.; writing-review and editing, M.S.E. and M.E.-M.; visualization, F.S.A. and K.M.A.; project administration, M.S.E. and M.E.-M.; funding acquisition, F.S.A. and K.M.A. All authors have read and agreed to the published version of the manuscript.

Funding: This research received no external funding.

Data Availability Statement: The data was mentioned along the paper.

Acknowledgments: This Research was supported by Taif University Researchers Supporting Project Number (TURSP-2020/217), Taif University, Taif, Saudi Arabia.

Conflicts of Interest: The authors declare no conflict of interest.

\section{References}

1. Jehhan, A.; Mohamed, I.; Eliwa, M.S.; Al-Mualim, S.; Yousof, H.M. The two-parameter odd Lindley Weibull lifetime model with properties and applications. Int. J. Stat. Probab. 2018, 7, 57-68.

2. Eliwa, M.S.; El-Morshedy, M.; Ibrahim, M. Inverse Gompertz distribution: Properties and different estimation methods with application to complete and censored data. Ann. Data Sci. 2019, 6, 321-339. [CrossRef]

3. Arshad, R.M.I.; Chesneau, C.; Jamal, F. The odd Gamma Weibull-geometric model: Theory and applications. Mathematics 2019, 7, 399. [CrossRef]

4. ZeinEldin, R.A.; Chesneau, C.; Jamal, F.; Elgarhy, M. Statistical properties and different methods of estimation for type I half logistic inverted Kumaraswamy distribution. Mathematics 2019, 7, 1002. [CrossRef]

5. ZeinEldin, R.A.; Chesneau, C.; Jamal, F.; Elgarhy, M. Different estimation methods for type I half-logistic Topp-Leone distribution. Mathematics 2019, 7, 985. [CrossRef]

6. Klakattawi, H.S. The Weibull-Gamma Distribution: Properties and Applications. Entropy 2019, 21, 438. [CrossRef]

7. Al-babtain, A.A.; Elbatal, I.; Yousof, H.M. A new flexible three-parameter model: Properties, Clayton copula, and modeling real data. Symmetry 2020, 12, 440. [CrossRef]

8. Yadav, A.S.; Goual, H.; Alotaibi, R.M.; Ali, M.M.; Yousof, H.M. Validation of the Topp-Leone-Lomax model via a modified Nikulin-Rao-Robson goodness-of-fit test with different methods of estimation. Symmetry 2020, 12, 57. [CrossRef]

9. Bantan, R.A.; Jamal, F.; Chesneau, C.; Elgarhy, M. Type II Power Topp-Leone generated family of distributions with statistical inference and applications. Symmetry 2020, 12, 75. [CrossRef]

10. Marshall, A.W.; Olkin, I. A new method for adding a parameter to a family of distributions with application to the exponential and Weibull families. Biometrika 1997, 84, 641-652. [CrossRef]

11. Zografos, K.; Balakrishnan, N. On families of Beta- and generalized Gamma-generated distributions and associated inference. Stat. Methodol. 2009, 6, 344-362. [CrossRef] 
12. Afify, A.Z.; Emrah, A.; Alizadeh, M.; Ozel, G.; Hamedani, G.G. The odd exponentiated half-logistic-G family: Properties, characterizations and applications. Chil. J. Stat. 2017, 8, 65-91.

13. El-Morshedy, M.; Eliwa, M.S. The odd flexible Weibull-H family of distributions: Properties and estimation with applications to complete and upper record data. Filomate 2019, 33, 2635-2652. [CrossRef]

14. Alizadeh, M.; Afify, A.Z.; Eliwa, M.S.; Sajid, A. The odd log-logistic Lindley-G family of distributions: Properties, Bayesian and non-Bayesian estimation with applications. Comput. Stat. 2019, 35, 281-308. [CrossRef]

15. El-Morshedy, M.; Eliwa, M.S.; Afify, A.Z. The odd Chen generator of distributions: Properties and estimation methods with applications in medicine and engineering. J. Natl. Sci. Found. Sri Lanka 2020, 48, 113-130.

16. Eliwa, M.S.; El-Morshedy, M.; Ali, S. Exponentiated odd Chen-G family of distributions: Statistical properties, Bayesian and non-Bayesian estimation with applications. J. Appl. Stat. 2020, 1-27. [CrossRef]

17. Tahir, M.H.; Hussain, M.A.; Cordeiro, G.M.; El-Morshedy, M.; Eliwa, M.S. A New Kumaraswamy Generalized Family of Distributions with Properties, Applications, and Bivariate Extension. Mathematics 2020, 8, 1989. [CrossRef]

18. Altun, E.; Korkmaz, M.Ç.; El-Morshedy, M.; Eliwa, M.S. The extended gamma distribution with regression model and applications AIMS Math. 2021, 6, 2418-2439. [CrossRef]

19. Altun, E.; El-Morshedy, M.; Eliwa, M.S. A new regression model for bounded response variable: An alternative to the beta and unit-Lindley regression models. PLOS ONE 2021, 16, e0245627. [CrossRef] [PubMed]

20. EL-Morshedy, M.; Alshammari, F.S.; Tyagi, A.; Elbatal, I.; Eliwa, M.S. Bayesian and frequentist inferences on a new probability generator with applications in engineering. Entropy 2021, 23, 446. [CrossRef]

21. Handique, L.; Chakraborty, S.; Eliwa, M.S.; Hamedani, G.G. Poisson Transmuted-G Family of Distributions: Its Properties and Applications. Pak. J. Stat. Oper. Res. 2021, 17, 309-332. [CrossRef]

22. Keller, A.Z.; Kamath, A.R. Reliability analysis of machine tools. Reliab. Eng. 1982, 3, 449-473. [CrossRef]

23. Abouammoh, A.M.; Alshingiti, A.M. Reliability of generalized inverted exponential distribution. J. Stat. Comput. Simul. 2009, 79, 1301-1315. [CrossRef]

24. Louzada, F.; Ramos, P.L.; Nascimento, D. The inverse Nakagami-m distribution: A novel approach in reliability. IEEE Trans. Reliab. 2018, 67, 1030-1042. [CrossRef]

25. Ramos, P.L.; Mota, A.L.; Ferreira, P.H.; Ramos, E.; Tomazella, V.L.; Louzada, F. Bayesian analysis of the inverse generalized gamma distribution using objective priors. J. Stat. Comput. Simul. 2021, 91, 786-816. [CrossRef]

26. Chang, G.J.; Cui, L.; Hwang, F.K. Reliabilities of Consecutive-K Systems; Kluwer Academic: Dordrecht, The Netherlands, 2009.

27. Nadarajah, S.; Bakouch, H.S.; Tahmasbi, R. A generalized Lindley distribution. Sankhya B 2011, 73, 331-359. [CrossRef]

28. Mahmoud, M.R.; Mandouh, R.M. On the transmuted Frechet distribution. J. Appl. Sci. Res. 2013, 9, 5553-5561.

29. Lawless, J.F. Statistical Models and Methods for Lifetime Data; John Wiley and Sons: New York, NY, USA, 2003. 